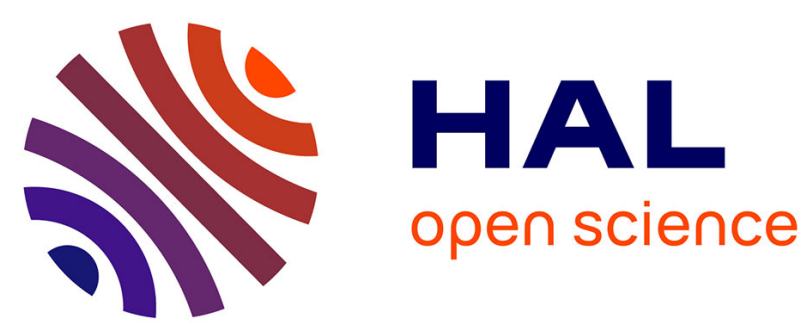

\title{
HOW TO CREATE EMPATHY AND \\ UNDERSTANDING: NARRATIVE ANALYTICS IN AGENT-BASED MODELING
}

Saikou Diallo, Christopher Lynch, Krzysztof Rechowicz, Grégory Zacharewicz

\section{- To cite this version:}

Saikou Diallo, Christopher Lynch, Krzysztof Rechowicz, Grégory Zacharewicz. HOW TO CREATE EMPATHY AND UNDERSTANDING: NARRATIVE ANALYTICS IN AGENT-BASED MODELING. 2018 Winter Simulation Conference (WSC), Dec 2018, Gothenburg, Sweden. pp.1286-1297, 10.1109/WSC.2018.8632267 . hal-02301955

\section{HAL Id: hal-02301955 \\ https://hal.science/hal-02301955}

Submitted on 10 Feb 2020

HAL is a multi-disciplinary open access archive for the deposit and dissemination of scientific research documents, whether they are published or not. The documents may come from teaching and research institutions in France or abroad, or from public or private research centers.
L'archive ouverte pluridisciplinaire HAL, est destinée au dépôt et à la diffusion de documents scientifiques de niveau recherche, publiés ou non, émanant des établissements d'enseignement et de recherche français ou étrangers, des laboratoires publics ou privés. 


\title{
HOW TO CREATE EMPATHY AND UNDERSTANDING: NARRATIVE ANALYTICS IN AGENT-BASED MODELING
}

\author{
Saikou Y. Diallo \\ Christopher J. Lynch \\ Krzysztof J. Rechowicz \\ Virginia Modeling, Analysis, and Simulation \\ Center, Old Dominion University \\ 1030 University Blvd. \\ Suffolk, VA 23322, USA
}

\author{
Gregory Zacharewicz \\ Laboratoire IMS UMR CNRS 5218 \\ University of Bordeaux \\ 351 Cours de la Libération \\ 33405 Talence cedex, FRANCE
}

\begin{abstract}
In this paper we propose a different approach for interacting and analyzing agent-based models. The approach relies on creating empathy and understanding between physical agents in the physical world (people) and artificial agents in the simulated world (simulated agents). We propose a simulated empathy framework (SEF) in which artificial agents communicate directly with physical agents through verbal channels and social media. We argue that artificial agents should focus on the communication aspects between these two worlds, the ability to tell their story in a compelling way, and to read between the lines of physical agents speech. We present an implementation of the SEF and discuss challenges associated with implementing the framework in an artificial society.
\end{abstract}

\section{INTRODUCTION}

Agent based Models (ABM) are difficult to follow and understand during execution. This is partly due to the fact that they are constructed bottom-up so that aggregate behavior can emerge from behaviors and interactions of individual agents within a shared environments (Epstein and Axtell 1996; Axelrod 1997; Epstein 1999). As a result, agents with only a small set of simple rules can lead to interesting and complex behaviors at the system level (Schelling 1971; Gilbert and Troitzsch 2005). The state of the art in analyzing agent-based models consists of 1) using charts, graphs, and other visual aids to convey information about agents or the model or 2) making batch runs to collect data for further analysis by other means. However, even for simple models, it is challenging to understand and track the internal state of agents and the impact of interactions with other agents. In cases where we claim emergence, it is difficult to provide a satisfactory explanation that starts at the agent level and culminates at the emergent behavior because most agent-based platforms do not offer a means for social interactions with individual agents during execution or for obtaining a meaningful story from those agents during or after execution in the form of history.

Storytelling and narratives are an effective medium for communicating simulation events, interactions, and results to non-experts by raising interest, comprehension, and engagement (Dahlstrom 2014). Storytelling has used games as a medium for years (Manney 2008) and has been explored as a promising avenue to assist in model conceptualization and communication between team members during design and implementation (Padilla et al. 2017). Agent history can be conveyed either 1) chronologically as news, 2) from the vantage point of a single actor, or 3) from a global point of view (Axelrod 1997). Simulations can be rerun to experience alternative histories, compare histories, and view the effects of altering the model's parameters on the resulting history. This can assist in identifying patterns within the model or inequalities among the agents. Traces track the characteristics of agents or the model over time to determine if the logic is correct and the simulation produces believable values (Epstein and Axtell 1996; Balci 1998; Kemper and 
Tepper 2005; Gore et al. 2017). However, the large volumes of data created through trace validation is difficult to analyze and can be burdensome to interpret (Courdier et al. 2002; Xiang et al. 2005). Conveying narrative results of simulation runs can be too lengthy to convey with appropriate detail since descriptions of one or more simulation runs' histories can require a significant amount of space (Axelrod 1997).

In this paper, we propose a conceptual switch to distinguish between physical agents who are people in the real world and artificial agents who are entities in the simulation world. In this conceptual arrangement, the goal is to create meaningful interactions between these two worlds during runtime to generate mutual understanding or empathy. Agents in the real world empathize with agents in the simulation world by experiencing their stories and their environment whereas agents in the simulation world empathize with agents in the real world by understanding their intent and providing them with the information they need. To that end, we propose a Simulated Empathy Framework (SEF) where physical agents can have personal and social interactions with artificial agents. The SEF relies on storytelling and narratives to create empathy and increase understanding between the two agent types. The balance of the paper is organized as follows: Section 2 presents background information on the state of the art in analyzing agent-based models; Section 3 describes the proposed SEF, a simple implementation, and a discussion; and we conclude in Section 4.

\section{BACKGROUND}

Agent-based Modeling allows for the rules at the individual level to produce system level behaviors (Epstein 1999; Ferber 1999); however, agent based models do not generally have built-in approaches for collecting and tracing agents throughout runtime. Current approaches for searching for individual level emergent behaviors generally focus on graphically tracking some attributes of an agent (Courdier et al. 2002; Lynch et al. 2017) or analytically searching the behavior space of an agent to discover interesting or relevant behaviors (Diallo et al. 2016; Gore et al. 2017). Both techniques utilize aspects of trace validation to collect information on agents and aggregate behaviors to provide insight into a modeling question. The results obtained largely focus on aggregate outcomes from the simulation's populations or the aggregated outcomes collected from multiple runs. Both approaches are powerful in providing partial explanations of aggregate behaviors through correlations, structural equation modeling, or statistical debugging. However, they lack the ability to provide satisfactory explanations of lifetime choices of agents.

Several factors hinder our ability to comprehend and explain an agent-based model. The most prevalent factor results from a conflicting perception that known macro level behaviors being modeled may be well understood but the mechanisms that lead to these behaviors may not be known (Bonabeau 2002; Takadama et al. 2008). Some expected behaviors and types of interactions among the agents within that system may also be known or are assumed to be understood. Muddling this understanding is that 1) modelers may have incorrect, incomplete, or contradictory knowledge about the system; 2) there is disagreement among stakeholders on how to address the problem or over which problem to solve (Lynch et al. 2014); 3) there are conflicting model characteristics that present verification and validation challenges (Lynch and Diallo $2015)$; 4) there are challenges resulting from simplifying the set of agent behaviors; 5) there are difficulties in examining the combinations and sequences of events leading to system level outcomes (Gore et al. 2017); and 6) there are challenges due to model components changing over time (Sargent 1996).

Visualization can provide clear insight on why simulations behaves in unexpected ways. Visualization enhances the experience of model execution by graphically representing parameter levels, distributions, network relationships, and interdependencies (Wenzel et al. 2003). This is accomplished in part by placing the simulation in context by co-locating different types of data within the simulation to gather explanations (Sokolowski and Garcia 2016). Animation provides the ability to observe how the system functions and helps to communicate results (Rohrer 2000). Some of the more commonly used techniques for visual insight include scatter plots, histograms, box plots (Sargent 1996), bar charts, icon-based displays, dense pixel displays (Keim 2002), data maps, and time-series plots (Forrester 1961). These techniques utilize datasets dependent upon size, type, or dimensionality (Keim 2002) for creating representations. Vernon-Bido et al. (2015) identify poor visualization choices and misrepresenting model components as potential visualization downfalls by adding additional levels of complexity to the model. 


\section{Diallo, Lynch, Rechowicz, and Zacharewicz}

Several efforts exist to provide interfaces for collecting, analyzing, and interpreting ABM data. Xiang et al. (2005) present a validation process for analyzing a natural organic matter model by using traces, graphs, charts, and model-to-model comparisons. Graphical charts test if the produced data curves match the expected normal distribution curve. This approach is suitable for inspecting population level statistics. Courdier et al. (2002) use the Geamas Virtual Laboratory (GVL) tool to collect traces of an ABM for analyzing animal wastes management. They collect sets of messages exchanged or historical accountings of one or more agents. Visualization tools filter information based on specific characteristics and identify successful agent negotiation interactions. The GVL tool becomes unwieldy over several dozen agents.

Lynch et al. (2017) explore the use of heat maps and spatial plots to visually represent the aggregate behaviors or aggregate distributions of agents throughout their environment during runtime. This approach to convey agent data helps illuminate pockets of the population that are grouping at specific geographic locations within the environment dependent upon their internal characteristics. They provide an example that visually compares the spatial distribution of obese agents (based on each individual's weight) against the spatial distribution of fast food restaurants within the environment.

Statistical debugging has also been utilized for analyzing simulation data. Initial applications of predicated-level statistical debugging are explored within software engineering (Gore et al. 2011; Gore and Reynolds Jr 2012) and later applied to simulations (Gore et al. 2015; Diallo et al. 2016). This method requires users to specify success and fail criteria within the execution and then collect input and output values generated during the simulation run. These values are then compared against the success/fail criteria in order to identify suspicious input or output values which are the parameters, behaviors, or agents commonly associated with failing cases (Gore et al. 2011; Gore et al. 2015).

Gore and Diallo (2013) apply statistical debugging at the individual level to identify unexpected behaviors within a model of obesity. Diallo et al. (2016) use statistical debugging at the model level to identify unexpected outcomes within an agent-based model of the Ballistic Missile Defense System. This approach uses input/output comparisons of numerous runs to identify input configurations that consistently lead to suspicious outcomes and allowed for an efficient examination of 1723 simulation runs. Diallo et al. (2016) present a V\&V Calculator to examine trace data and provide statistical measures on characteristics that are likely contributors to model behaviors. Gore et al. (2017) extend this process to provide a structured approach for specifically examining traces of agent and/or model characteristics from agent-based models.

Beyond visual inspection, audial feedback provides feedback on individual level events. Rauterberg and Styger (1994) use sound feedback to increase communication, improve plant operator performance, and affect aspects of mood to increase positivity in plant operators. They observed that sound was adapt at providing feedback on simultaneous events, a finding that is directly applicable to providing information on agent populations during execution. Sound based feedback has been explored for its ability to assist in collaboration by providing a non-intrusive awareness of ongoing events and encouraging joint problem solving (Gaver 1991). Gaver et al. (1991) found that sound was beneficial for separately examining discrete processes within a simulation or to cumulatively examine the simulation as a whole.

Narratives and storytelling are used for conceptualizing and sharing models. Padilla et al. (2017) examine the use of narrative and storyboarding for conceptualizing and designing simulations. Games, as a form of storytelling, are used to teach simulation concepts ranging from teaching Discrete Event Simulation in general (Padilla et al. 2016) to teaching the concepts of delay and feedback within System Dynamics (Sterman 1989). However, to our knowledge, we are unaware of studies where artificial agents provide narratives or stories back to the physical agents from their own perspectives.

With an increasing variety of social media platforms and access to information posted directly by people about their daily lives, key events, and their likes and dislikes, there exists possibilities for connecting simulations directly into the "human" component of data. Padilla et al. (2014) and Kavak et al. (2018) explore the use of social media data in simulations as sources of input data, for validation and calibration, for recognizing mobility patterns of a population, and identifying communication patterns and agent relations. Gore et al. (2015) show that tweet information allows for approximating individual level measurements of wellbeing through people self-reporting on healthy eating and regular exercise in the same 
way that could have been obtained through a survey. Padilla et al. (2018) use tweets to identify individuallevel tourist visit patterns and sentiment. These types of information sources can provide new routes towards developing population-based behaviors and rules within agent based models.

Approaches reviewed in this section rely on 1) observable execution at runtime, 2) large numbers of runs, and, 3) the ability to interpret visual and/or statistical analyses of data. The aims of these approaches are to identify key events, successes, and failures during execution and to illuminate the conditions contributing to these occurrences. None of these approaches aim to tell the overall story of the model or its agents over time to create a holistic understanding of the situation in the model.

\section{SIMULATED EMPATHY FRAMEWORK}

We propose a framework for connecting artificial agents to real world agents for the purpose of generating empathy. The primary function of this framework is to create a connection layer that connects the real world with the simulated world which can be achieved through digital senses (Rechowicz et al. 2018). The model and physical agent exist in the real world; artificial agents exist in a state of an executing simulation within the simulated world, and the SEF seeks to bridge these worlds during runtime. The SEF connects observers with a simulation's execution by allowing the simulated agents to create narratives based on their experiences and provide those back to the observers. Figure 1 shows the SEF and highlights specific question areas, the questions we seek to address within those areas, and design challenges for maintaining consistency between physical and artificial agents.

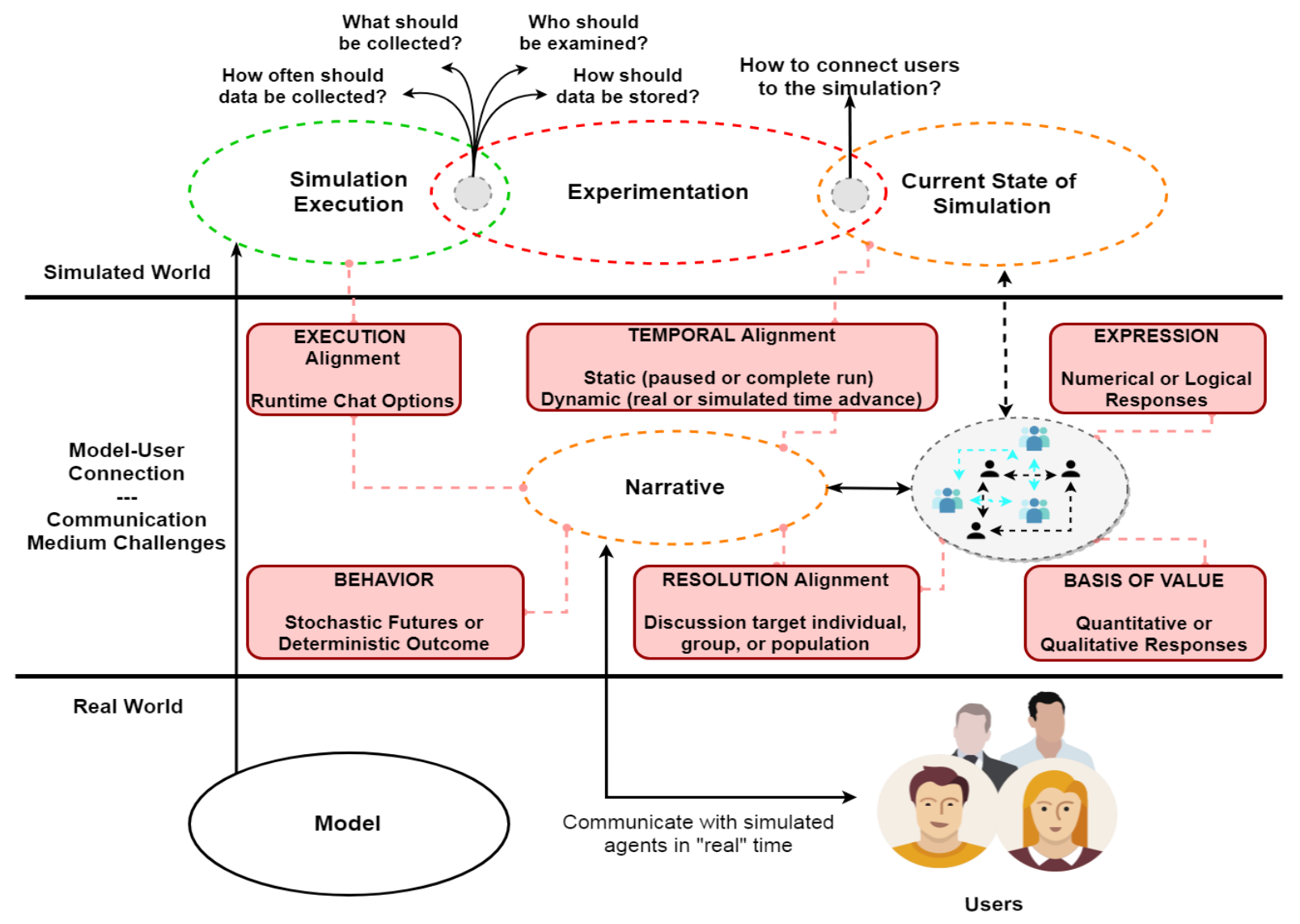

Figure 1: Narrative-Conversation Communication Framework. 


\section{Diallo, Lynch, Rechowicz, and Zacharewicz}

To facilitate physical-to-simulated communication, the SEF uses a model-user connection layer to connect the real world with the simulated world. Instead of exploring the physical space within the simulation (i.e. through code, visualizations, statistics, etc.), the SEF uses narrative communication to describe the current state of the simulation. Agents' personal experiences are conveyed within their narratives to create an empathetic path between the simulated agent and the real observer at the point in time (real and simulated) that the communication takes place. Three main components comprise the SEF:

- Physical component: The physical component includes physical agents (people) and connected objects. The physical component can be augmented, virtual, or extended and encompasses communication interfaces through any channel (physical, verbal, social media, etc.);

- Communication and intelligence component: The communication and intelligence component is comprised of processing units that transform and transfer inputs from physical agents to artificial agents and vice versa. This component includes natural language processes, reasoning, and analytical frameworks, such as IBM Watson and Amazon's Skills; and

- Artificial component: The artificial component holds the simulated world and all relevant data and communication means. This includes the states of entities, lists of events, as well as the communication means that links and create relationships from the artificial to the physical agents (i.e. people).

The SEF creates a number of unique challenges for maintaining temporal consistencies across worlds during communication. In this paper we focus on the communication and intelligence component. To begin, we present design considerations and specifications to facilitate physical to artificial agent communication.

\subsection{Design Considerations}

Creating a framework to allow physical agents to communicate with artificial agents during execution raises several considerations for aligning real world perceptions with simulation constraints. These considerations are examined with respect to aligning communication options with six dimensions of model characteristics as outlined in (Lynch and Diallo 2015) adapted from two taxonomies for classifying simulation tools in parallel and distributed systems (Sulistio et al. 2004). The dimensions are time representation; basis of value; behavior; expression; resolution; and execution. These categories place unique challenges on the ability to connect physical and artificial agents through narrative storytelling and communication.

- Time representation considers temporal alignment between the real time advancement of the physical agents and the simulated time advancement of the artificial agents. The model can potentially be paused and simulation time can advance at faster or slower rates than real time. Should communication only occur at static points in time when the simulation is paused to maintain a consistent simulation state during communication? If the model executes dynamically, should time advancement change to advance at real time during communication; should it pause until the communication is complete; should it continue running at its original speed? What happens if an agent dies in the simulation while it is part of a conversation? Can physical agents only communicate with currently living agents or should narratives be attainable from agents that have passed away during the simulation run?

- Basis of value considers the difference between qualitative and quantitative style response from the agents. Different narratives can be provided by an agent simply by discussing age in terms of a numeric value, such as "I am 87 years old", versus a qualitative response, such as "I am old". Both styles of response are appropriate depending on the type of communication being offered by the physical agent. However, providing quantitative values in a response that does not warrant that level of specificity will be out of place and may be off-putting to the physical agent and vice-versa.

- Behavior considers whether deterministic (always generating the same output for a given input) or stochastic (generating potentially many outputs for a given input) simulation outputs are generated. 
However, an expanded perspective is required for agent-person communication. If communication occurs using a simulation that has already completed execution, then the simulation can now only be considered a deterministic run, even if the simulation is stochastic, because the agents can only communicate on what already happened during execution. New or different events cannot occur even if the physical agent has the ability to roll back the simulation time and communicate with agents in their past. To ensure that stochastic behaviors are maintained, multiple runs can be conducted and agent histories can be aggregated from multiple runs to narrate collections of possible futures.

- Expression deals with how artificial agents communicate their attribute values, events that they experience, and decisions that they make. Many of these considerations are dependent upon agents' attributes' data types. For instance, communicating an age value represented as a decimal can be handled differently than a blindness state represented as true or false.

- Resolution requires considering whether physical agents can communicate with artificial agents, groups of artificial agents or populations of artificial agents. How wide is the circle of inclusion for the artificial agent Self only? Spouse? Family? Friends? Co-workers?

- Execution requires considering how communication channels to the agents interact with the execution of the simulation. Should agents' internal logic be updated to place them into explicit "communication" states when communicating with a real person? Without proper temporal alignment, this approach could lead to an agent lagging behind the other agents by years before the chat ends and the normal behavior of the agent resumes. Alternatively, not putting the agent into a paused state could result in the death of the agent prior to the end of the conversation.

To achieve an end state where agents are able to respond to real requests and generate informative narratives about their lives, we need to shift from the traditional visual and statistical approaches only for tracing and communicating agent behaviors to adding a narrative based approach for conveying agent histories, key events, and current states. The combination of all of these questions reveal that our framework needs to provide communication mediums, categories of communication styles, different aggregation levels of agents for communication, and considerations for past and present agent timelines.

\subsection{Communicating Through the SEF}

Three primary questions remain to be addressed. What forms of communications can take place? What communication mediums are available? What types of responses are the artificial agents able to provide?

We classify communication styles under two broad categories: solicited, initiated by the physical agent; and unsolicited, initiated by the artificial agent. Solicited communications are assumed to be at least loosely structured in a question and answer format. These communications result in either the return of a quantitative or qualitative style response from the agent and can relate to agent attributes, locations, states, connections, or other personal information. Unsolicited communications are provided periodically by the artificial agent and can pertain to the its history or key life events. History communications are time-event pairs of information traced over time, such as employment geographic location. Life events are the high value events occurring to the artificial agent directly or with its network connections, such as a friend having a child. Table 1 provides the communication styles contained in our framework.

We categorize human-to-simulation communication mediums as either direct or indirect. Direct occurs face-to-face, through a connected console, or through the simulation's interface. Indirect includes social media platforms. Face-to-face involves the physical agent visually observing the artificial agent using a computer monitor, augmented reality, or virtual reality. A connected console allows for the selection of and communication with agents within the simulation using a device which is not running the simulation. The simulation interface facilitates communication occurs through the same device that executes the simulation. Social media platforms involve communicating directly to the intended target but through an intermediary textual interface, such as the physical or artificial agent's twitter handle. 
Diallo, Lynch, Rechowicz, and Zacharewicz

Table 1: Physical-Artificial agent communication styles.

\begin{tabular}{|l|c|l|}
\hline \multicolumn{1}{|c|}{ Interaction Type } & \multicolumn{1}{|c|}{$\begin{array}{c}\text { Physical Agent } \\
\text { Solicitation Requirement }\end{array}$} & \multicolumn{1}{|c|}{ Agent Target } \\
\hline $\begin{array}{l}\text { Quantitative Question } \\
\text { and Answer }\end{array}$ & Solicited & $\begin{array}{l}\text { Attribute, state, event, location, and } \\
\text { network information }\end{array}$ \\
\hline $\begin{array}{l}\text { Qualitative Question } \\
\text { and Answer }\end{array}$ & Solicited & $\begin{array}{l}\text { Attribute, state, event, location, and } \\
\text { network information }\end{array}$ \\
\hline History & Unsolicited & Time-event information \\
\hline Life Event & Unsolicited & $\begin{array}{l}\text { Key event information pertaining to self } \\
\text { and network connections }\end{array}$ \\
\hline
\end{tabular}

Direct communication mediums provide a more familiar computer-oriented interface for communicating with digital entities. This style of communication operates on more immediate feedback style responses that the indirect mediums. Ideally, if the physical agent can see the artificial agent and ask a question, then the physical agent expects a response on a similar timeframe to asking a physical person a question. Indirect mediums do not necessarily share the immediate feedback approach as questions posed through social media are not always seen immediately upon being sent. Benefits of using indirect communication mediums include the ability to initiate communication at any time as the artificial agent does not have to be available, removing the spatial constraint of the physical agent needing to remain near the simulation console in order to receive responses from the artificial agents, and the ability to pose questions from any mobile connected device. Figure 2 provides the division of communication mediums.

To enable communication along both of these mediums we implement three options: chat windows; message based connection; and Twitter (Twitter 2018). Chat windows provide the means of communicating when accessing the simulation directly. The User Datagram Protocol (UDP) protocol (Postel 1980) provides communication access for face-to-face and connected consoles as these utilize external devices that connect to the simulation. We gain access to Twitter through its freely available developer API to provide the ability for artificial agents to receive and respond to messages sent by people from any location at any time.

Once the communication route is established, the artificial agent still needs to formulate responses. We categorize three narrative resolution levels: atomic; composite; and aggregate. Atomic responses contain information only pertaining to the selected artificial agent. Composite responses contain information about the artificial agent's connected networks or groups. Aggregate responses pertain to the entire artificial agent population or model level occurrences. The selection of response categories and communication mediums do not restrict the communication styles available. Figure 3 provides the narrative resolution levels.

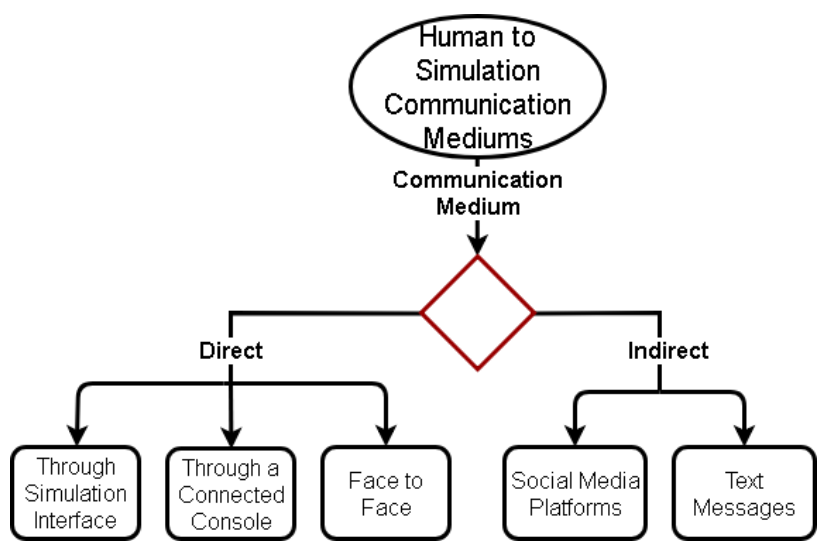

Figure 2: Physical-Artificial communication mediums.

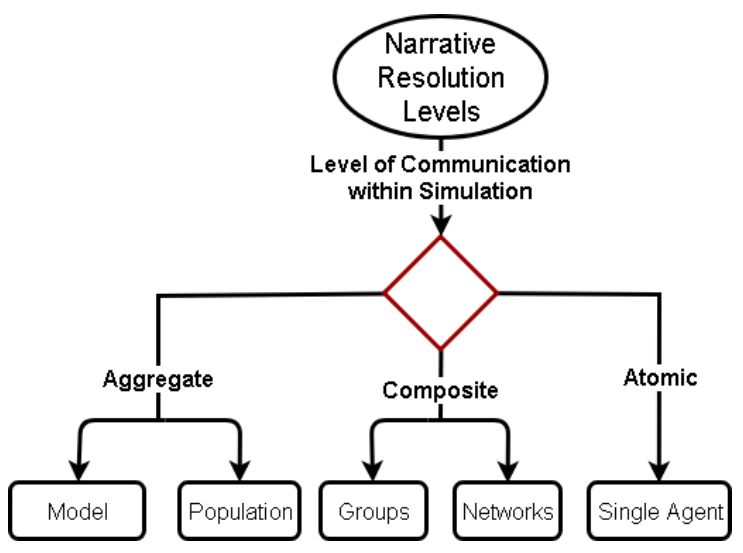

Figure 3. Artificial agent narrative resolution levels. 
We provide an initial use case of our framework using a baseline model named Simla. There is a minimal set of rules governing this agent based model. Agents and workplaces are distributed randomly within an environment. Agents can belong to three types of social networks: family; employment (coworkers); and online (non-family, non-coworker). Family networks can span multiple generations and include spouses, (grand)children, and (grand)parents. Over time, agents are seeking jobs and marriage. Agent histories are recording significant events, which include marriage, change in employment status, births (self and people directly connected through one of the networks), and deaths of any connected agents.

For communicating stories, we conduct an experiment using only indirect communication through the Twitter social media platform using its API (Twitter 2018). Artificial agents can communicate three categories of updates through Twitter: communication; sentiment; and network. For communication, artificial agents have the ability to provide updates on their status based on their significant life events that have occurred and post their updates to the twitter handle @VirginiaMASC. Artificial agents also have the ability to respond to tweets mentioning this handle. The culmination of tweets posted during a run provides the story of an agent's life. Sentiment options include the ability to like and retweet tweets. We have not implemented a specific framework for selecting how to like or retweet a tweet. For an exploration into how and why people retweet, see (Boyd et al. 2010). Network options involve the ability for the artificial agent to expand or adjust its real-world communication network by following new physical agents or blocking and muting physical agents. While the network options specify for blocking, muting, and following physical agents, the reverse of these actions, unblocking, unmuting, and unfollowing, are also possible. Figure 4 provides an overview of the available communication options within this use case.

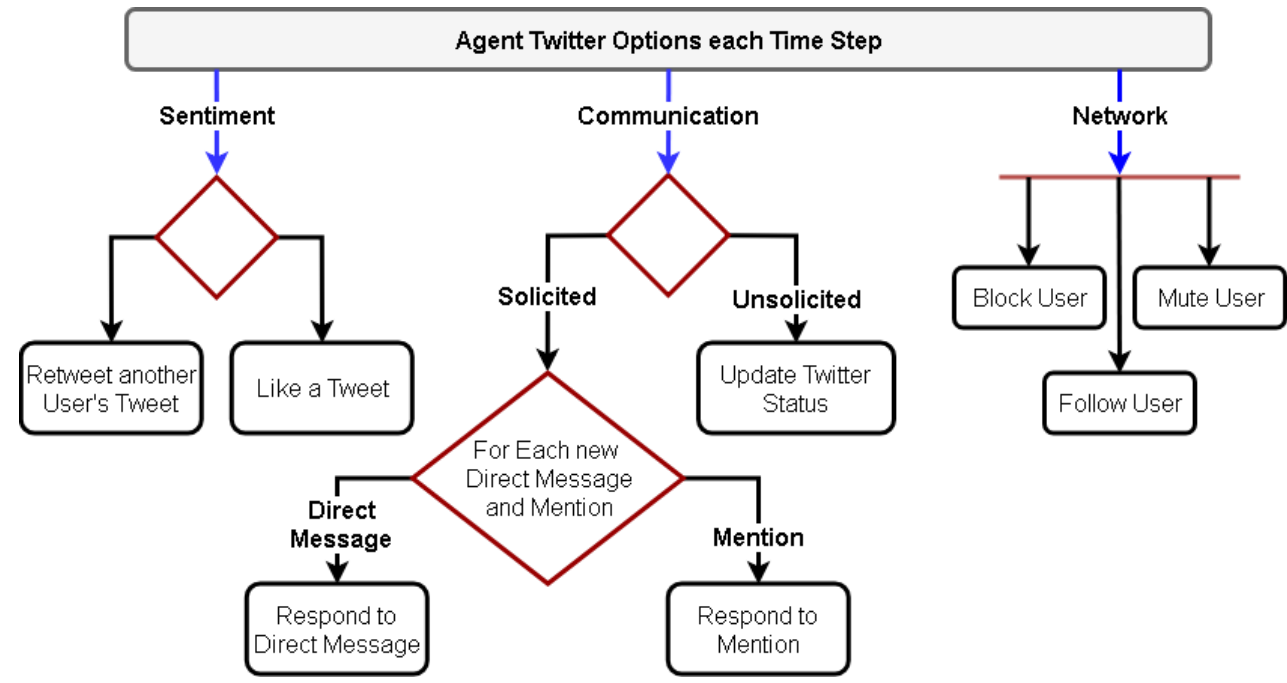

Figure 4: Artificial agent communication options through Twitter.

We provide a comparative example of the narrative provided by an artificial agent from a sample run using a combination of visualizations, tweets, chat windows, and UDP communication. We use pie charts to convey the annual status of the population. Tweets provide the story of key events pertaining to the agent while the chat and UDP communication options provide direct communication between real people and artificial agents. Figure 5 provides the outcome from the sample run. With this dashboard, a physical agent can follow the artificial society and its individual agents. The physical agent can explore connections between aggregate level phenomena and agents' tweets. For instance, a large set of tweets about artificial agents losing jobs might be an indicator of an impending rise in intolerance at the societal level. A lack of rise in intolerance might lead the physical agent to seek out one of the recently unemployed agents to engage in conversation. Physical agents may then gain a better understanding of the life of an artificial agent which can lead them to formulate additional questions and further improve communication and empathy. 


\section{Diallo, Lynch, Rechowicz, and Zacharewicz}

Our current testing has focused solely on communicating with single artificial agents. Future work involves extending our implementation to communicate with artificial agents at different resolution levels. For instance, newscaster style artificial agents could be used for communicate key events within an area or pertaining to specific social networks. A method for associating artificial agents' tweets to their corresponding runs, with originating time steps, is needed to maintain traceability to the simulation.

Chat Window: Week 4

Conversation with Agent: Index 1945, ID 3978: Amanda
You:Hi
Amanda: Hello.
You: Are you employed?
Amanda: I am current1y a STUDENT.
You: Where do you live?
Amanda: I live in Ealing_Neighbourhood.
You: Do you have any children?
Amanda: I do not have any children.
You: How Old are you?
Amanda: I am 30 years old.
You: Do you have any siblings?
Amanda: I have 1 siblings.
You: How are your parents?
Amanda: My father is NOT EMPLOYED and my mother is NOT EMPLOYED.

Direct Communication Using UDP: Week 4

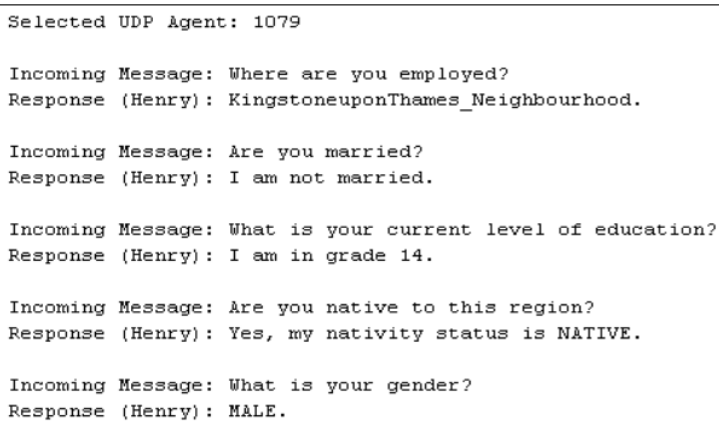

Tweets

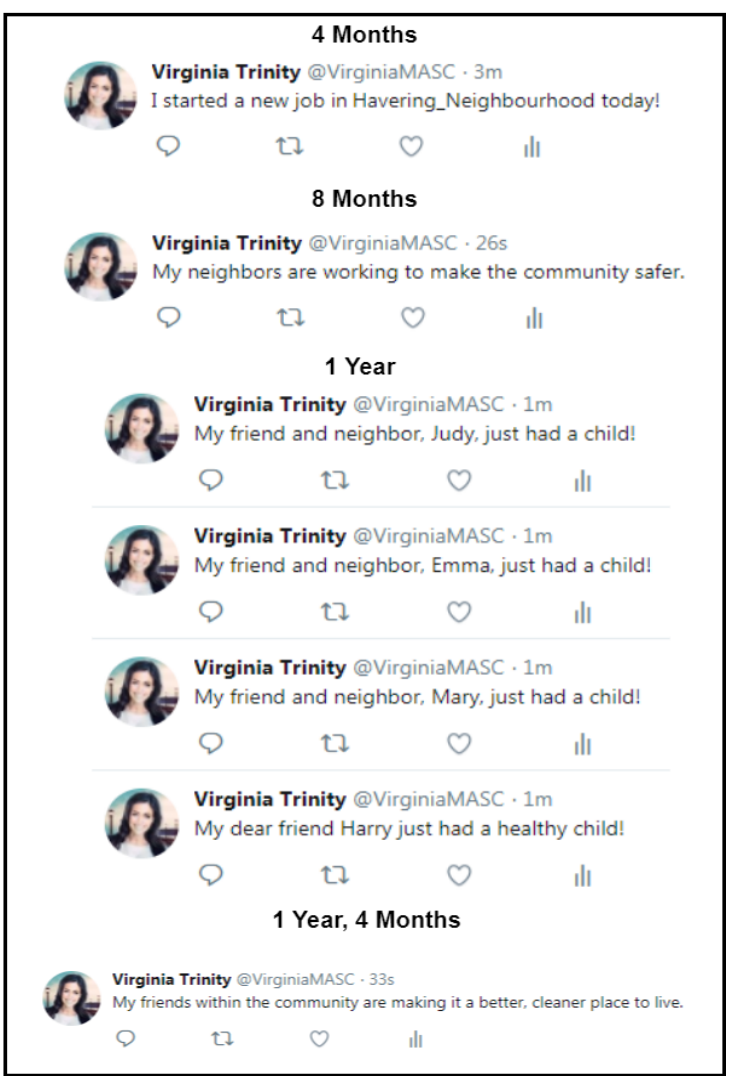

My friends within the community are making it a better, cleaner place to live. t] 0

Visualization

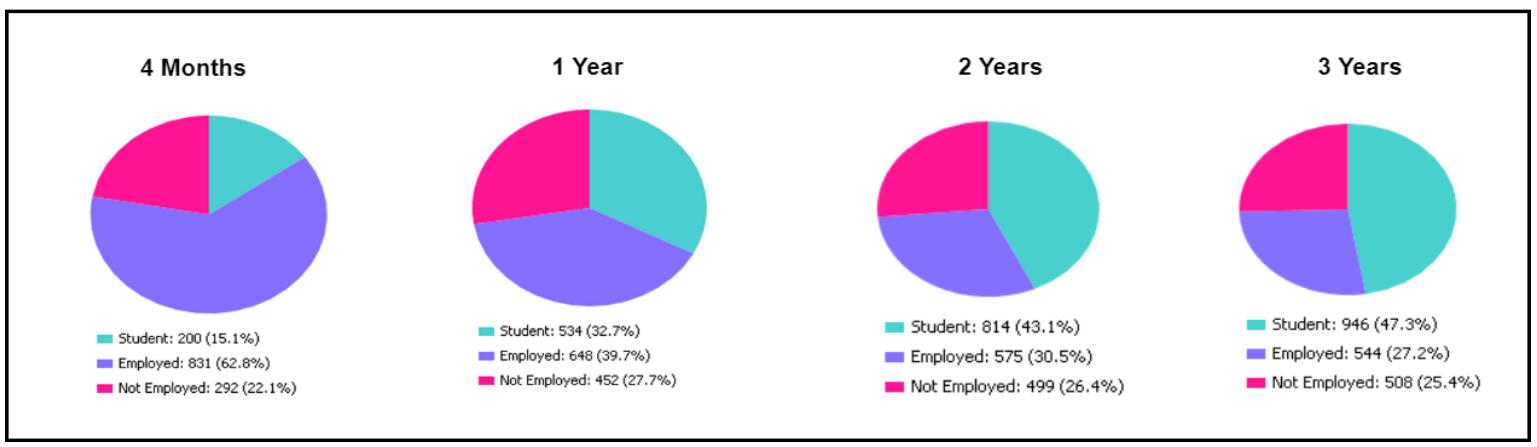

Figure 5: Comparative snapshot of a sample model run using pie charts, text, and tweets.

\section{CONCLUSION}

A number of model initialization considerations for tracing artificial agent progressions arise when creating a communication architecture. Should artificial agents report on events that occurred in their past prior to the start of the simulation run? For instance, artificial agents have the ability to communicate when their 
children were born throughout execution because information pertaining to that event is stored at the time that the birth occurs; however, should artificial agents that initialize with children start with a collection of stored events corresponding to their children, their own employment start dates, etc., where applicable?

The SEF is only the beginning of a vast research area that aims at "breaking the wall" between artificial and physical worlds. The SEF presents challenges at the computational, social, cognitive, and philosophical levels. We have begun to apply it to art, archeology, humanities, policy, and decision support. The success of a SEF approach relies on transdisciplinary teams working together to advance our understanding on the human condition. The SEF calls for a different form of interaction between human and artificial agents. Advances in connected objects, extended reality, and digital senses can be leveraged natively to that end.

\section{REFERENCES}

Axelrod, R. 1997. "Advancing the Art of Simulation in the Social Sciences". Complexity 3 (2):16-22.

Balci, O. 1998. "Verification, Calibration, and Testing". In Handbook of Simulation, edited by J. Banks, 335-393. New York, NY: Wiley and Sons, Inc.

Bonabeau, E. 2002. "Agent-Based Modeling: Methods and Techniques for Simulating Human Systems". Proceedings of the National Academy of Sciences of the United States of America 99 (Suppl 3):72807287.

Boyd, D., S. Golder, and G. Lotan. 2010. "Tweet, Tweet, Retweet: Conversational Aspects of Retweeting on Twitter". In Proceedings of the 43rd Hawaii International Conference on System Sciences (HICSS).

Courdier, R., F. Guerrin, F. H. Andriamasinoro, and J.M. Paillat. 2002. "Agent-Based Simulation of Complex Systems: Application to Collective Management of Animal Wastes". Journal of Artificial Societies and Social Simulation 5 (3):1-17.

Dahlstrom, M. F. 2014. "Using Narratives and Storytelling to Communicate Science with Nonexpert Audiences". Proceedings of the National Academy of Sciences 111 (Supplement 4):13614-13620.

Diallo, S. Y., R. Gore, C. J. Lynch, and J. J. Padilla. 2016. "Formal Methods, Statistical Debugging and Exploratory Analysis in Support of System Development: Towards a Verification and Validation Calculator Tool". International Journal of Modeling, Simulation, and Scientific Computing 7 (1):1-22.

Epstein, J. M. 1999. "Agent - Based Computational Models and Generative Social Science". Complexity 4 (5):41-60.

Epstein, J. M., and R. Axtell. 1996. Growing Artificial Societies: Social Science from the Bottom Up. 1st ed. Cambridge, MA: The MIT Press

Ferber, J. 1999. Multi-Agent Systems: An Introduction to Distributed Artificial Intelligence. 1st ed. Boston, MA: Addison-Wesley Reading

Forrester, J. W. 1961. Industrial Dynamics. 1st ed. Cambridge, MA: The MIT Press

Gaver, W. W. 1991. "Sound Support for Collaboration". In Proceedings of the Second European Conference on Computer-Supported Cooperative Work ECSCW'91, edited by L. Bannon, M. Robinson, and K. Schmidt, 293-308. Springer Science and Business Media.

Gaver, W. W., R. B. Smith, and T. O'Shea. 1991. "Effective Sounds in Complex Systems: The Arkola Simulation". In Proceedings of the SIGCHI Conference on Human factors in Computing Systems, 8590. New York, NY: ACM.

Gilbert, N., and K. Troitzsch. 2005. Simulation for the Social Scientist. 2nd Ed. New York: McGraw-Hill.

Gore, R., and S. Y. Diallo. 2013. "The Need for Usable Formal Methods in Verification and Validation". In Proceedings of the 2013 Winter Simulation Conference, edited by R. Pasupathy et al., 1257-1268. Piscataway, New Jersey: IEEE.

Gore, R., S. Y. Diallo, and J. J. Padilla. 2015. "You Are What You Tweet: Connecting the Geographic Variation in America's Obesity Rate to Twitter Content". PloS one 10 (9):1-16.

Gore, R., C. J. Lynch, and H. Kavak. 2017. "Applying Statistical Debugging for Enhanced Trace Validation of Agent-Based Models". Simulation 93 (4):273-284. 
Gore, R., and P. F. Reynolds Jr. 2012. "Reducing Confounding Bias in Predicate-Level Statistical Debugging Metrics". In Proceedings of the 34th International Conference on Software Engineering (ICSE), 463-473. Piscataway, NJ: IEEE.

Gore, R., P. F. Reynolds Jr, and D. Kamensky. 2011. "Statistical Debugging with Elastic Predicates". In Proceedings of the 26th IEEE/ACM International Conference on Automated Software Engineering, 492-495. Piscataway, NJ: IEEE.

Gore, R., P. F. Reynolds Jr, D. Kamensky, S. Y. Diallo, and J. J. Padilla. 2015. "Statistical Debugging for Simulations". ACM Transactions on Modeling and Computer Simulation (TOMACS) 25 (3):1-26.

Kavak, H., D. Vernon-Bido, and J. J. Padilla. 2018. "Fine-Scale Prediction of People's Home Location Using Social Media Footprints". In 2018 International Conference on Social Computing, BehavioralCultural Modling, \& Prediction and Behavior Representation in Modeling and Simulation, 1-6.

Keim, D. A. 2002. "Information Visualization and Visual Data Mining". IEEE Transactions on Visualization and Computer Graphics 8 (1): 1-8.

Kemper, P., and C. Tepper. 2005. "Trace Based Analysis of Process Interaction Models". In Proceedings of the 2005 Winter Simulation Conference, edited by M. E. Kuhl et al., 427-436. Piscataway, New Jersey: IEEE.

Lynch, C. J., and S. Diallo. 2015. "A Taxonomy for Classifying Terminologies That Describe Simulations with Multiple Models". In Proceedings of the 2015 Winter Simulation Conference, edited by L. Yilmaz et al.,1621-1632, Piscataway, New Jersey: IEEE.

Lynch, C. J., H. Kavak, R. Gore, and D. Vernon-Bido. 2017. "Identifying Unexpected Behaviors of AgentBased Models through Spatial Plots and Heat Maps". In Swarmfest 2017: 21 st Annual Meeting on Agent Based Modeling and Simulation, 1-14.

Lynch, C. J., J. J. Padilla, S. Y. Diallo, J. A. Sokolowski, and C. M. Banks. 2014. "A Multi-Paradigm Modeling Framework for Modeling and Simulating Problem Situations". In Proceedings of the 2014 Winter Simulation Conference, edited by A. Tolk et al., 1688-1699, Piscataway, New Jersey: IEEE.

Manney, P. J. 2008. "Empathy in the Time of Technology: How Storytelling Is the Key to Empathy". Journal of Evolution and Technology 19 (1):51-61.

Padilla, J. J., S. Y. Diallo, H. Kavak, O. Sahin, and B. Nicholson. 2014. "Leveraging Social Media Data in Agent-Based Simulations". In Proceedings of the 2014 Spring Simulation Multiconference - Annual Simulation Symposium, 221-228. San Diego, CA: SCS.

Padilla, J. J., H. Kavak, C. J. Lynch, R. J. Gore, and S. Y. Diallo. 2018. "Temporal and Spatiotemporal Investigation of Tourist Attraction Visit Sentiment on Twitter". PloS One 13 (6):1-20.

Padilla, J. J., C. J. Lynch, H. Kavak, S. Y. Diallo, R. Gore, A. Barraco, and B. Jenkins. 2016. "Using Simulation Games for Teaching and Learning Discrete-Event Simulation". In Proceedings of the 2016 Winter Simulation Conference, edited by T. M. K. Roeder et al., 3375-3385, Piscataway, New Jersey: IEEE.

Padilla, J. J., C. J. Lynch, H. Kavak, S. Evett, D. Nelson, C. Carson, and J. del Villar. 2017. "Storytelling and Simulation Creation". In Proceedings of the 2017 Winter Simulation Conference, edited by W. K. V. Chan et al., 4288-4299. Piscataway, New Jersey: IEEE.

Postel, J. 1980. "User Datagram Protocol". Document number RFC 768.

Rauterberg, M., and E. Styger. 1994. "Positive Effects of Sound Feedback During the Operation of a Plant Simulator". In Human-Computer Interaction, edited by B. Blumenthal, J. Gornostaev, and C. Unger, 35-44. Berlin, Heidelberg: Springer.

Rechowicz, K. J., S. Y. Diallo, H. M. Garcia, J. B. Shull, and B. Cvijetic. 2018. "Making Digital Sense[S]: Fundamentals". In Proceedings of the 2018 Spring Simulation Multiconference - ANSS, 1-11. SCS.

Rohrer, M. W. 2000. "Seeing Is Believing: The Importance of Visualization in Manufacturing Simulation". In Proceedings of the 2000 Winter Simulation Conference, edited by J. A. Joines et al., 1211-1216, Piscataway, New Jersey: IEEE. 
Sargent, R. G. 1996. "Some Subjective Validation Methods Using Graphical Displays of Data". In Proceedings of the 1996 Winter Simulation Conference, edited by J. M. Charnes et al., 345-351, Piscataway, New Jersey: IEEE.

Schelling, T. 1971. "Dynamic Models of Segregation". Journal of Mathematical Sociology 1 (2):143-186.

Sokolowski, J. A., and H. M. Garcia. 2016. "The Significance of Modeling and Visualization". In The Digital Patient: Advancing Healthcare, Research, and Education, edited by C. D. Combs, J. A. Sokolowski, and C. M. Banks, 33-48. Hoboken, NJ: John Wiley \& Sons, Inc.

Sterman, J. D. 1989. "Modeling Managerial Behavior: Misperceptions of Feedback in a Dynamic Decision Making Experiment". Management Science 35 (3):321-339.

Sulistio, A., C. S. Yeo, and R. Buyya. 2004. "A Taxonomy of Computer-Based Simulations and Its Mapping to Parallel and Distributed Systems Simulation Tools". Software: Practice and Experience 34(7):653.

Takadama, K., T. Kawai, and Y. Koyama. 2008. "Micro-and Macro-Level Validation in Agent-Based Simulation: Reproduction of Human-Like Behaviors and Thinking in a Sequential Bargaining Game". Journal of Artificial Societies and Social Simulation 11 (2):1-17.

Twitter. 2018. "Https://Developer.Twitter.Com". Twitter, Inc. https://developer.twitter.com/en.html. Accessed 11.04.2018.

Vernon-Bido, D., A. J. Collins, and J. A. Sokolowski. 2015. "Effective Visualization in Modeling \& Simulation". In Proceedings of the 2015 Spring Simulation Multi-Conference - Annual Simulation Symposium, 33-40. San Diego, CA: SCS.

Wenzel, S., J. Bernhard, and U. Jessen. 2003. "Visualization for Modeling and Simulation: A Taxonomy of Visualization Techniques for Simulation in Production and Logistics". In Proceedings of the 2003 Winter Simulation Conference, edited by S. Chick, et al., 729-736, Piscataway, New Jersey: IEEE.

Xiang, X., R. Kennedy, G. Madey, and S. Cabaniss. 2005. "Verification and Validation of Agent-Based Scientific Simulation Models". In Proceedings of the 2005 Spring Simulation Multi-Conference Agent-Directed Simulation Symposium, 47-55. San Diego, CA: SCS.

\section{AUTHOR BIOGRAPHIES}

SAIKOU Y. DIALLO is a Research Associate Professor at the Virginia Modeling, Analysis, and Simulation Center (VMASC) where he serves as the head of the Simulated Empathy lab. He is also an adjunct Professor of Modeling, Simulation, and Visualization Engineering at Old Dominion University (ODU). He received his $\mathrm{MS}$ and $\mathrm{PhD}$ in Modeling and Simulation from ODU. His email address is sdiallo@odu.edu and his web page is http://www.odu.edu/vmasc/research/simulated-empathy.html.

CHRISTOPHER J. LYNCH is a Senior Project Scientist at VMASC at ODU. He received his M.S. in Modeling and Simulation (M\&S) from ODU in 2012 and a B.S. in Electrical Engineering from ODU in 2011. He is currently pursuing a Ph.D. in M\&S from ODU with a focus on using sound to verify simulations. His email address is cjlynch@odu.edu and his web page is http://www.odu.edu/directory/people/c/clync008.

KRZYSZTOF J. RECHOWICZ is a research assistant professor VMASC at ODU. He received his MS in Mechanics and Machine Construction (2006) from the Warsaw University of Technology and a PhD in M\&S (2012) from ODU. His research interests focus on bridging the gap between the physical and digital world through sensory and reality augmentation. He is currently the Digital Senses Lab lead. His email address is krechowi@odu.edu and his web page is http://www.odu.edu/vmasc/research/digital-senses.html.

GREGORY ZACHAREWICZ is an Associate Professor with Habilitation at the University of Bordeaux (France). His research interests include distributed simulation, semantics aspects, model driven approaches, discrete event M\&S, and enterprise modeling. He received his MS (2002) and PhD (2006) in Modelling and Simulation from Aix-Marseille University. His email address is gregory.zacharewicz@u-bordeaux.fr and his web page is https://www.ims-bordeaux.fr/en/annuaire/5124-zacharewicz-gregory\#display-misc. 